\title{
Surgical Therapy for a Solitary Form of Hepatic Epithelioid Hemangioendothelioma: A Long-Term Survival Case
}

\author{
Nobu Oshima Hiroaki Terajima Ryo Hosotani \\ Department of Surgery, Kobe City Medical Center General Hospital, Kobe, Japan
}

\section{Key Words}

Epithelioid hemangioendothelioma - Liver · Surgical resection · Long survival

\begin{abstract}
Hepatic epithelioid hemangioendothelioma (HEHE) is a rare neoplasm of vascular origin. The clinical presentation of HEHE is variable, and the therapeutic criteria are still unclear since its natural history is unpredictable. A 53-year-old woman was admitted to our hospital because she had a $2.5-\mathrm{cm}$ diameter nodule in the segment $\mathrm{V}$ of the liver. She had undergone segmental hepatectomy for solitary HEHE in segment VII 10 years before. There had been no recurrence for the 10 years after the first operation. The tumor was diagnosed as a new lesion of HEHE by percutaneous needle liver biopsy, and thereafter repeated hepatectomy was performed. HEHE seems to be resistant to chemotherapy and radiotherapy. Either surgical resection or orthotopic liver transplantation is generally recommended as a curative treatment for this disease. However, HEHE tends to be detected in multiple lesions, and localized disease is rare. Therefore, the chance of resection is very low. Some reports do not recommend local resection because of early aggressive tumor spread even after curative resection. We herein demonstrate a rare case of HEHE in a patient who underwent repeated hepatectomy for a solitary lesion and who survived for 17 years. It is concluded that surgical resection is one of the most effective treatments for a solitary form of HEHE.
\end{abstract}

\section{Introduction}

Hepatic epithelioid hemangioendothelioma (HEHE) is a rare neoplasm of vascular origin with an unpredictable clinical course and prognosis. Epithelioid hemangioendothelioma (EHE) was first described by Weiss and Enzinger [1] in 1982. EHE usually occurs in soft tissues and bones, but rarely in the liver. HEHE is generally considered as a low-grade malignancy. Some cases were cured only by surgical resection while, in contrast, others were systemically metastatic and fatal disease at the time of 
diagnosis $[2,3]$. Due to the variability of the natural course, the therapeutic strategy remains to be determined. We herein report a patient who had two successful operations for a solitary lesion of HEHE and who survived for 17 years.

\section{Case Report}

The patient was an asymptomatic 53-year-old woman. She had been diagnosed with a hepatic neoplasm in 1991, but had refused to be operated on due to personal matters. However, she was willing to attend follow-up. During a follow-up period of three years, this hepatic tumor had grown from 2 to $3 \mathrm{~cm}$ in diameter. In August 1994, she underwent segmental hepatectomy of segment VII. The tumor was histopathologically diagnosed as HEHE. After a follow-up period of 10 years after the first operation, in September 2004, routine ultrasonography revealed a well-defined and low-echoic nodule $2.5 \mathrm{~cm}$ in diameter in the anterior segment of the liver.

Laboratory data of liver function test were within normal range: aspartate aminotransferase, $18 \mathrm{IU} / \mathrm{l}$; alanine aminotransferase, $17 \mathrm{IU} / \mathrm{l}$; total bilirubin, $0.2 \mathrm{mg} / \mathrm{dl}$; alkaline phosphatase, $135 \mathrm{IU} / \mathrm{l}$; $\gamma$-glutamyl-transpeptitase, $13 \mathrm{IU} / \mathrm{l}$; prothrombin activity, $105.7 \%$. The data of tumor markers, such as carcinogenic embryonic antigen (CEA), carbohydrate antigen 19-9 (CA19-9), alpha-fetoprotein and protein induced by vitamin $\mathrm{K}$ absence or antagonist (PIVKAII) was also within normal range.

Computed tomography showed a solitary and low-density nodule in segment $\mathrm{V}$ of the liver. There were, however, no extrahepatic lesions in the lung, mediastinum, skin or soft tissue. The peripheral rim of the tumor, but not the center, was enhanced by contrast media in the early phase. Likewise, in the late phase, the intensity at the peripheral rim of the tumor was enhanced to a similar level as in the adjacent normal liver parenchyma, but again it did not change in the central part of the tumor (fig. 1a, b, c). T1-weighted axial magnetic resonance imaging showed a low signal intensity of the tumor, while a T2-weighted image showed a high signal. Celiac angiography showed a hypovascular tumor, but only the tumor margin was enhanced (fig. 1d). No extrahepatic neoplasm was detected on extensive examination, including colonoscopy and gastroscopy.

Based on these findings, the tumor was suspected to be a new recurrent lesion of HEHE or cholangiocellular carcinoma. Immunohistochemical staining of the tumor tissue obtained using percutaneous ultrasonography-guided needle liver biopsy revealed that the tumor cells were positive for CD34 and factor VIII antigen, negative for CEA and CA19-9, and as a result the tumor was diagnosed as a recurrence of HEHE.

The patient underwent subsegmental hepatectomy of segment $\mathrm{V}$ of the liver. The postoperative course was uneventful. A yellowish-white and non-encapsulated tumor approximately $2.5 \mathrm{~cm}$ in diameter and of rather hard consistency was macroscopically observed on the surface of the liver (fig. 2). Histopathological examination demonstrated that the cellular components were dominant in the peripheral region, while hyaline depositions were dominant in the central region. The tumor consisted of spindle-shaped cells and signet-ring-cell-like structures with intracytoplasmic lumina. Epithelioid cells were round in shape and had an eosinophilic cytoplasm and vesicular nuclei. Immunohistochemical staining revealed that epithelioid tumor cells were positive for CD34, factor VIII antigen and vimentin, and negative for CEA and CA19-9 (fig. 3). These findings were compatible with previous EHE reports.

There has been no evidence of recurrent tumors for 4 years after the second hepatectomy. The patient has survived for 17 years after detection of the first HEHE.

\section{Discussion}

HEHE is a rare hepatic tumor of vascular origin [1-4]. EHE was classified as a malignant vascular tumor according to the $2002 \mathrm{WHO}$ classification, but its natural course and prognosis are still unpredictable. Most HEHE patients are reported to present with nonspecific symptoms and are often asymptomatic with the tumor being detected coincidentally by imaging studies. Laboratory data are not usually useful for diagnosis of HEHE. Serum levels of tumor markers such as CEA, CA19-9 and alpha-fetoprotein 
remain within normal limit. As the disease advances, however, clinical symptoms such as general malaise and abdominal discomfort appear, and laboratory data, including liver function test, may become worse $[2,3]$. In this report, the patient was symptom-free and all laboratory data were within normal range. On ultrasonography, the echogenicity of the lesion was variable. The lesions most frequently show a hypoechogenic pattern compared with the adjacent liver parenchyma, and are occasionally isoechoic with a hypoechoic rim [5].

Among the imaging studies, computed tomography is the most sensitive imaging system for defining the extent of the tumor. Furui et al. [6] described two different types of HEHE, (1) nodular type and (2) diffuse type. They demonstrated that the nodular lesions were an earlier form of HEHE which later changed into diffuse lesions. The nodular type was observed in only $11.1 \%$ of patients at initial presentation, and $66 \%$ of cases had multiple lesions [2]. In the present case, however, the solitary lesion developed twice at the interval of 10 years, and the patient underwent two curative segmental hepatectomies for this solitary form of HEHE. HEHE most frequently shows a multifocal growth pattern at the time of diagnosis, therefore this kind of manifestation is very rare. Whether a solitary form is a temporary manifestation of multiple nodular form is still unknown, since very few reports regarding the solitary form of HEHE have been described previously. It might be an earlier stage of the multiple nodular form.

Histological and immunohistochemical examinations are most useful for definitive diagnosis of HEHE. In immunohistochemical staining, the neoplastic cells are positive for factor VIII, CD31, and CD34, which are the endothelial cell makers [1-3]. In a recent study by Makhlouf et al. [3], core needle biopsy was performed in $22 \%$ of 137 cases. However, it should be noted that there is a risk of peritoneal seeding in image-guided core biopsy because of the usual location of HEHE at the periphery of the liver. Therefore, it is necessary to choose a transhepatic route of puncture through the normal hepatic parenchyma, not directly into the tumor, if feasible.

As to conservative treatment, various kinds of therapy for patients with HEHE have been reported, but it is difficult to assess the effectiveness of each therapy and to decide on the therapeutic strategy for HEHE because of the rarity and unpredictable behavior of this neoplasm. The effectiveness of chemotherapy and radiotherapy is still not clear, but some authors have suggested that interferon $a-2 b$ is effective for HEHE [7]. In previously reported HEHE cases, there were several patients who remained alive for several years without any therapy [4]. Only one case report demonstrated spontaneous complete regression of HEHE without any treatment [8].

In contrast, surgical treatment such as curative liver resection and orthotopic liver transplantation (OLT) have frequently provided relatively satisfactory clinical results $[2,3,9,10]$. In a recent study by Mehrabi et al. [11], they reported that the overall survival rate of patients who underwent liver resection was longer than that of patients undergoing OLT. Surgical resection for localized HEHE seems to be accepted as a standard therapeutic strategy. However, they also reported that only $9.4 \%$ of 286 patients underwent liver resection. Therefore, the curative resection rate for HEHE is still relatively low [10] because of the multicentric or multifocal occurrence of the tumor. Ben-Haim et al. described aggressive recurrence after curative surgical resection for resectable lesion by imaging series [12]. The authors suspected that during hepatic regeneration, some agent, such as hepatotrophic growth factor, stimulates HEHE proliferation [12]. However, the majority of those cases were the multiple form of HEHE, not the solitary form as in our case. The patient has not experienced early aggressive recurrence after two liver resections. Although there are no randomized control studies 
on solitary or multiple lesion of HEHE because of its rarity, we consider that surgical resection may be a good option for patients with a solitary HEHE and no extrahepatic lesions.

It has recently been reported that OLT has provided a good outcome for the diffuse type of HEHE. Some reports revealed that OLT was effective for HEHE even with extrahepatic localization of the disease [13]. Prognosis of HEHE is significantly better in comparison with that of other hepatic malignancies. However, less is known about truly long-term (more than 5 years) outcomes. As mentioned above, some patients were well for several years without any therapy. Interestingly, it appears that tumor spread has no influence on prognosis $[2,12-14]$.

In conclusion, we recommend hepatic resection for a solitary form of HEHE without extrahepatic lesions. For the multiple nodular type of HEHE without extrahepatic lesions, the indication of hepatic resection should be carefully determined, even if technically possible. In order to establish a therapeutic algorithm for HEHE, further studies and case series are necessary. 


\begin{tabular}{r|l|l|l} 
Case Reports in & $\begin{array}{l}\text { Case Rep Gastroenterol 2009;3:214-221 } \\
\text { D0I: 10.1159/000227734 }\end{array}$ & Published online: August 28, 2009 & $\begin{array}{l}\text { O 2009 S. Karger AG, Basel } \\
\text { ISSN 1662-0631 } \\
\text { www.karger.com/crg }\end{array}$ \\
\hline
\end{tabular}

Fig. 1. Abdominal computed tomography findings showed a small low-density nodule with the rim enhanced in the early phase. a Plane. b Early phase. c Late phase. Celiac angiography showed a pale tumor stain with a relatively strong staining in the tumor margin $(\mathbf{d})$.
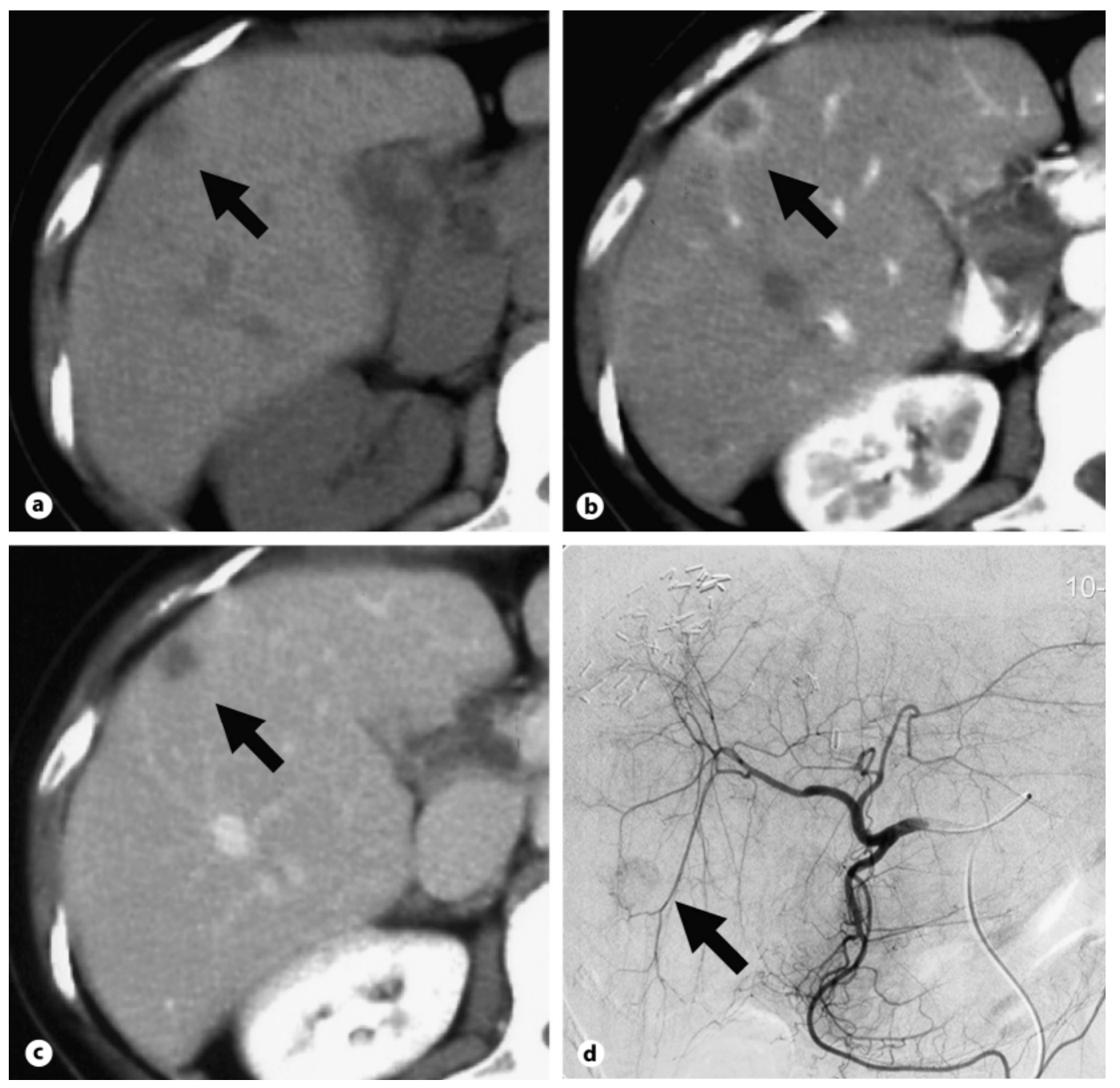


\begin{tabular}{r|l|l|l} 
Case Repports $/ \mathrm{h}$ & $\begin{array}{l}\text { Case Rep Gastroenterol 2009;3:214-221 } \\
\text { D01: 10.1159/000227734 }\end{array}$ & Published online: August 28, 2009 & $\begin{array}{l}\text { 2009 S. Karger AG, Basel } \\
\text { ISSN 1662-0631 } \\
\text { www.karger.com/crg }\end{array}$ \\
\hline
\end{tabular}

Fig. 2. The resected specimen showed a yellowish-white tumor with a moderately hard consistency $(\mathbf{a}, \mathbf{b})$.
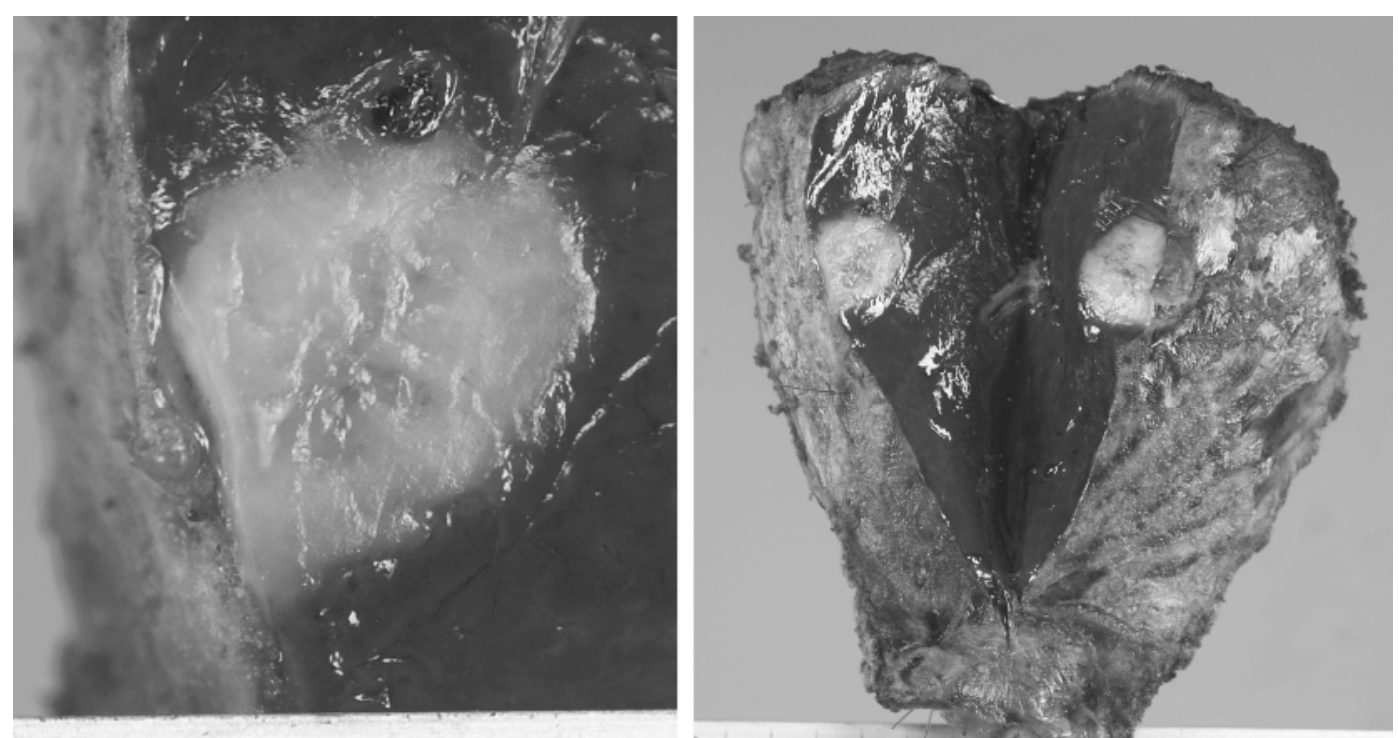

a

b 


\begin{tabular}{r|l|l|l} 
Case Reports in & $\begin{array}{l}\text { Case Rep Gastroenterol 2009;3:214-221 } \\
\text { D0I: 10.1159/000227734 }\end{array}$ & Published online: August 28, 2009 & $\begin{array}{l}\text { O 2009 S. Karger AG, Basel } \\
\text { ISSN 1662-0631 } \\
\text { www.karger.com/crg }\end{array}$ \\
\hline
\end{tabular}

Fig. 3. Histopathological findings demonstrated that cellular components were dominant in the peripheral region, while hyaline deposits were dominant in the central region (a), and that the tumor consisted of spindle-shaped cells and signet-ring-cell-like structures with intracytoplasmic lumina (b). Immunohistochemical staining showed that tumor cells were positive for CD34 (c) and factor VIII (d). a $\mathrm{H} \& \mathrm{E}, \times 20$. b H\&E, $\times 40$.
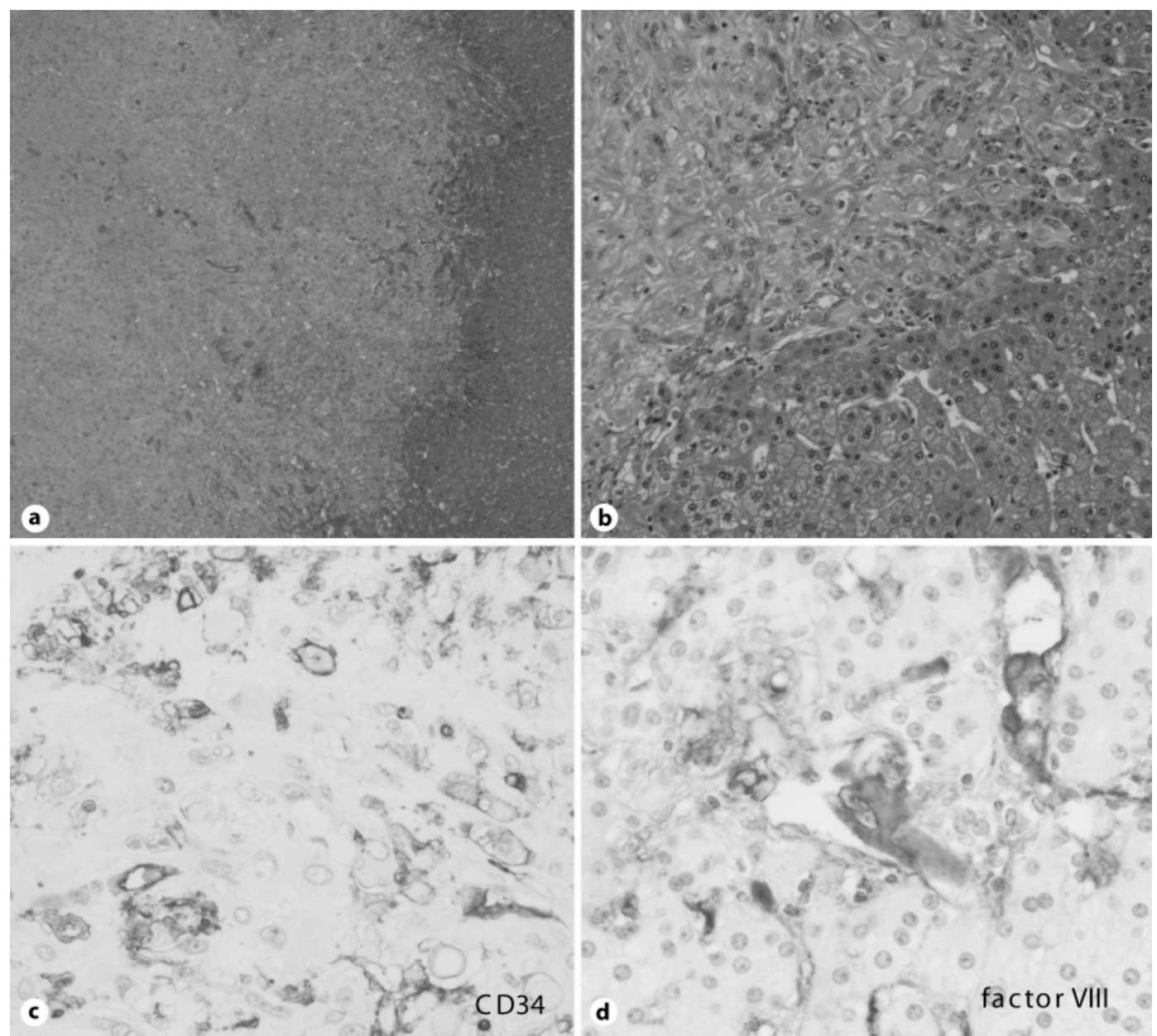


\section{References}

1 Weiss SW, Enzinger FM: Epithelioid hemangioendothelioma: a vascular tumor often mistaken for a carcinoma. Cancer 1982;50:970-981.

2 Lauffer JM, Zimmermann A, Krahenbuhl L, et al: Epithelioid hemangioendothelioma of the liver. A rare hepatic tumor. Cancer 1996;78:23182327.

-3 Makhlouf HR, Ishak KG, Goodman ZD: Epithelioid hemangioendothelioma of the liver: a clinicopathologic study of 137 cases. Cancer 1999;85:562-582.

-4 Ishak KG, Sesterhenn IA, Goodman ZD, et al: Epithelioid hemangioendothelioma of the liver: a clinicopathologic and follow-up study of 32 cases. Hum Pathol $1984 ; 15: 839-852$.

-5 Lyburn ID, Torreggiani WC, Harris AC, et al: Hepatic epithelioid hemangioendothelioma: sonographic, CT, and MR imaging appearances. AJR Am J Roentgenol 2003;180:1359-1364.

-6 Furui S, Itai Y, Ohtomo K, et al: Hepatic epithelioid hemangioendothelioma: report of five cases. Radiology 1989;171:63-68.

7 Galvao FH, Bakonyi-Neto A, Machado MA, et al: Interferon alpha-B and liver resection to treat multifocal hepatic epithelioid hemangioendothelioma: a relevant approach to avoid liver transplantation. Transplant Proc 2005;37:43544358 .

8 Otrock ZK, Al-Kutoubi A, Kattar MM, et al: Spontaneous complete regression of hepatic epithelioid haemangioendothelioma. Lancet Oncol 2006;7:439-441.

-9 Uchimura K, Nakamuta M, Osoegawa M, et al: Hepatic epithelioid hemangioendothelioma. J Clin Gastroenterol 2001;32:431-434.

10 Nagase M, Ryu M, Kinoshita T, et al: Epithelioid hemangioendothelioma of the liver. J Hepatobiliary Pancreat Surg 2000;7:443-447.

$\checkmark 11$ Mehrabi A, Kashfi A, Fonouni H, et al: Primary malignant hepatic epithelioid hemangioendothelioma: a comprehensive review of the literature with emphasis on the surgical therapy. Cancer 2006;107:2108-2121.

12 Ben-Haim M, Roayaie S, Ye MQ, et al: Hepatic epithelioid hemangioendothelioma: resection or transplantation, which and when? Liver Transpl Surg 1999;5:526-531.

13 Lerut JP, Orlando G, Sempoux C, et al: Hepatic haemangioendothelioma in adults: excellent outcome following liver transplantation. Transpl Int 2004;17:202-207.

14 Marino IR, Todo S, Tzakis AG, et al: Treatment of hepatic epithelioid hemangioendothelioma with liver transplantation. Cancer 1988;62:2079-2084. 